\title{
ULTRALOW-TEMPERATURE RESONANT GRAVITATIONAL WAVE DETECTORS, PRESENT STATE AND FUTURE PROSPECTS
}

\author{
G. Frossati
}

Kamerlingh Onnes Laboratory, Leiden University, PO Box 9506, 2300 AG Leiden, The Netherlands

\section{ABSTRACT}

Resonant gravitational wave detectors have evolved from the room temperature bars of J. Weber to the subKelvin bars of the Rome and Padua groups. Their strain sensitivity $h=\Delta L / L$ is now in the range of $10^{-19}$, thought to be enough to detect events like supernovae explosions within our Galaxy, events which are very rare. There is still room for improvement but in order to detect more frequent events, the future detectors must gain a factor 100 or more in $h$, thus $10^{4}$ in energy sensitivity. For this, spherical (or spheroidal) detectors are being designed which will be cooled to $50 \mathrm{mK}$ or less. Besides the higher planned sensitivity, a number of advantages relative to high directionality, the possibility of detecting both polarizations of the wave, and the possibility of putting upper limits on theories which predict scalar waves by measuring the monopole mode makes these antennas very attractive and an ideal complement to the interferometers. In the few-kilohertz range, low-cost spherical antennas can be built with sensitivities comparable to advanced interferometers working in narrow-band mode. $\odot 2003$ Published by Elsevier Ltd on behalf of COSPAR.

\section{INTRODUCTION}

Weber (1960) pioneered the experimental search for gravitational waves. The sensitive element of his resonance detector was a cylindrical aluminium bar $153 \mathrm{~cm}$ long and $66 \mathrm{~cm}$ in diameter. The bar was suspended by a wire in vacuum and was mechanically decoupled from its surroundings. Around its middle were attached piezoelectric strain transducers, which coupled into electronic circuits that were sensitive to the bar's fundamental end-to-end mode of oscillation. When a gravitational wave burst hits the bar perpendicular to the cylinder axis the space-time deformation $h=\Delta L / L$ that it causes is also seen by the bar. The resulting acceleration excites its fundamental mode with an initial amplitude that is given by $\Delta L=h L$.

The sensitivity of a detector is determined by the dominating noise sources in competition with the signal, primarily mechanical, acoustic and thermal noise. First, one has to reduce the acoustic noise reaching the antenna along the suspension (with the use of mass-spring systems) to levels well below those we expect to measure. The remaining noise is usually thermal noise and the electronic noise of the detector. The motion transducer is nowadays a resonant transducer, usually a small mass (or even two or three for multi-mode transducers) attached resonantly to the antenna. Because of the resonance the energy deposited will oscillate between the antenna and the detector and the displacement of the transducer is amplified by the square root of the ratio of the antenna to transducer masses. The detector mass causes a modulation of a static electric or magnetic field placed between the part of the transducer fixed to the antenna and the transducer oscillating mass. The resulting AC voltage is read by means of a sensitive SQUID having noise $k_{B} T_{N}=n h v$, where $v$ is the frequency of the detected signal. Commercial SQUIDs have $n \approx 1000-5000$ and the goal is to reach $n=$ 1 , the quantum limit. Wcbcr uscd piczoelectric ceramics placed at the center of his bar, that was kept at room 
temperature. A resonant gravitational wave antenna measures the Fourier transform $H(\omega)$ of the strain amplitude $h(t)$ defined as

$$
H(\omega)=\int_{-\infty}^{\infty} h(t) e^{i \omega t} d t
$$

(Note that a gravitational wave has two polarizations $h_{+}$and $h_{x}$ but we consider only one). Several hypotheses can be made about the spectral content of the gravitational wave. For the case of a burst of duration $\tau_{g} \sim 1 / v_{g}$, (with $v_{g}=\omega_{0} / 2 \pi$ ) corresponding to a flat spectrum from 0 to $v_{g}$ we can write $h(t) \sim$ $H\left(\omega_{0}\right) / \tau_{y}$ which in terms of the antenna parameters is written as

$$
h(t)=\frac{L}{v_{s}^{2} \tau_{g}} \sqrt{\frac{k_{B} T_{e f f}}{M}}
$$

$\nu_{s}$ is the sound velocity of the antenna material and $T_{e f f}$ is the effective temperature of the antenna and transducer given by

$$
T_{\mathrm{eff}} \approx \frac{T}{\beta Q}+2 T_{N}
$$

where $T$ is the thermodynamic temperature of the antenna, $\beta$ is a coupling parameter smaller than 1 for passive transducers, and $Q$ is the mechanical quality factor of the bar. $\beta$ measures the ratio of the energy transferred to the transducer to that deposited on the antenna. Bar antennas with single-mass transducers have $\beta \approx 10^{-3}$. Small values of $\beta$ mean also small bandwidth, typically a few $\mathrm{Hz}$ thus increasing $\beta$ is one of the most important goals together with increasing the sensitivity.

Weber's bar was at room temperature, had a mass of $1400 \mathrm{~kg}$, and a resonant frequency $f_{0}=1661 \mathrm{~Hz}$ for a sound velocity of $5460 \mathrm{~m} / \mathrm{s}$. Taking $\beta \approx 10^{-3}$ and $Q \approx 10^{5}$ (a typical value for aluminium) Eq. (3) gives $T_{e f f} \approx$ $3 \mathrm{~K}$, which when substituted in Eq. (2), gives $h \approx 10^{-17}$. If a signal comes from a gravitational wave it should simultaneously be observable by a second bar separated by a large distance from the first. Weber employed two identical experimental setups, one at the University of Maryland, the other at the Argonne National Laboratory near Chicago, $1000 \mathrm{~km}$ away. He recorded several coincident signals and claimed (Weber, 1969) evidence for observation of gravitational waves. These and subsequent observations by Weber were greeted with great excitement in the early 1970s, however, there was also growing skepticism as the observations implied that the strength of the gravitational waves was very much in excess of what was expected which is typically $h \approx 10^{-18}-10^{-19}$ for supernovae explosions in our Galaxy.

\section{PRESENT STATE OF BAR DETECTORS}

From Eq. (2) it is clear that the sensitivity of the bar detectors can be improved by increasing the mass and by lowering the temperature. Today, three decades after Weber's pioneering experiments, there are several operational bar detectors in different parts of the world all working at cryogenic temperatures (Table 1) and having a higher mass than Weber's bar. In Table 1 the sensitivity is given in $h=\Delta L / L$ for a millisecond burst. The theoretical sensitivy is derived from Eq. (2) for the quantum limit of $n=1$, a high $\beta \approx 0.05$ and $Q$ $\approx 10^{7}$ (except for NIOBE where we assume $\beta \approx 1$, because they use a parametric transducer, and $Q \approx 10^{8}$ ). 
Table 1: Present-day large cryogenic bar detectors

\begin{tabular}{|c|c|c|c|c|c|c|c|c|}
\hline name & site & $\begin{array}{c}\text { opera- } \\
\text { tional } \\
\text { since }\end{array}$ & $\begin{array}{c}\text { resonant } \\
\text { frequency } \\
(\mathrm{Hz})\end{array}$ & $\begin{array}{l}\text { resonator } \\
\text { mass }\end{array}$ & $\begin{array}{l}\text { tempe- } \\
\text { rature }\end{array}$ & $\begin{array}{c}\text { theoretical } \\
\text { sensitivity } \\
\text { (Q.L) }\end{array}$ & $\begin{array}{l}\text { experim. } \\
\text { sensitivity }\end{array}$ & reference \\
\hline EXPLORER & CERN & 1990 & $907-923$ & $2270 \mathrm{~kg} \mathrm{Al}$ & $2.5 \mathrm{~K}$ & $1.7 \times 10^{-20}$ & $6 \times 10^{-19}$ & $\begin{array}{l}\text { Astone et } \\
\text { al. }(1997)\end{array}$ \\
\hline ALLEGRO & Louisiana & 1991 & $897-920$ & $2300 \mathrm{~kg} \mathrm{Al}$ & $4.2 \mathrm{~K}$ & $2.2 \times 10^{-20}$ & $6 \times 10^{-19}$ & $\begin{array}{l}\text { Hamilton et } \\
\text { al. (1997) }\end{array}$ \\
\hline NIOBE & Perth & 1993 & 710 & $1500 \mathrm{~kg} \mathrm{Nb}$ & $5.7 \mathrm{~K}$ & $\sim 10^{-20}$ & $6 \times 10^{-19}$ & $\begin{array}{l}\text { Blair et } \\
\text { al. (1997) }\end{array}$ \\
\hline NAUTILUS & Rome & 1995 & $906-921$ & $2350 \mathrm{~kg} \mathrm{Al}$ & $95 \mathrm{mK}$ & $3.9 \times 10^{-21}$ & $4 \times 10^{-19}$ & $\begin{array}{l}\text { Astone et } \\
\text { al. (1997) }\end{array}$ \\
\hline AURIGA & Padua & 1995 & 920 & $2300 \mathrm{~kg} \mathrm{Al}$ & $100 \mathrm{mK}$ & $4 \times 10^{-21}$ & $4 \times 10^{-19}$ & $\begin{array}{l}\text { Cerdonio et } \\
\text { al. (1997) }\end{array}$ \\
\hline
\end{tabular}

Cryogenic operation, superconducting electronics, improved vibration isolation and increased acoustic $Q$ factors have contributed to a $10^{4}$-fold improvement in energy sensitivity over Weber's original antennas (a factor 100 in $h$ ). Coincidence experiments are being performed between various combinations of cryogenic bar detectors. So far, gravitational waves have not yet been detected.

\section{SPHERICAL GRAVITATIONAL WAVE DETECTORS}

It was recognized long ago (Forward, 1971) that a sphere is a very natural shape for a resonant-mass detector of gravitational waves. A free elastic sphere has five degenerate quadrupole modes of vibration, each of which is sensitive to a different combination of polarization and direction of gravity waves: both the source direction (modulo $\pi$ ) and wave polarization can be determined from the relative amplitudes of these quadrupole modes. Cylindrical Weber-bar detectors have only one useful quadrupole mode. A spherical detector has a much larger cross section for gravitational waves than a cylindrical Weber bar of the same material and resonant frequency. Firstly, the sphere has a mass which is much larger than that of a typical cylinder of the same size, material and resonant frequency. Secondly, the sphere is sensitive to both polarizations of gravitational waves, and thirdly, the sphere's sensitivity to gravitational waves is isotropic, so its cross-section is not reduced (by a factor of $\sim 5$ ) when averaged over all possible directions (Wagoner and Paik, 1977). The total energy cross-section improvement over a typical bar antenna is about a factor of 80 (Coccia et al. ,1995).

A nearly spherical antenna, called a Truncated Icosahedral Gravitational wave Antenna (TIGA), was suggested and worked out in detail by Merkowitz and Johnson (1995). TIGA's outer surface is composed of 32 flat surfaces ( 20 hexagons and 12 pentagons). Secondary resonators would be attached to 6 pentagonal faces. One design assumes an aluminium sphere with a radius of $1.3 \mathrm{~m}$, giving a mass of $25.1 \mathrm{t}$ and a resonant frequency of $1 \mathrm{kHz}$. At that frequency the sensitivity would be $10^{-23} / \sqrt{\mathrm{Hz}}$, one order of magnitude better than the first-generation LIGO detector. Plans to actually build TIGA were, however, not followed up.

Another proposal (Frossati, 1997) for a spherical detector was the Gravitational Radiation Antenna In Leiden (GRAIL). GRAIL was envisaged as a sphere with a radius of $1.5 \mathrm{~m}$ made of CuAl with a density of $8 \mathrm{~g} / \mathrm{cm}^{3}$ (total mass: $113 \mathrm{t}$, effective mass: $24.43 \mathrm{t}$, resonant frequency: $770 \mathrm{~Hz}$ ). Since at least 5 transducers are required for the 5 resonant quadrupole modes, and an additional detector for the other modes to act as veto, the actual $Q$ factor of the detector will be degraded from the bare very high intrinsic $Q$ factors. This has been seen with single-transducer bars made from $\mathrm{Al} 5056$ or $\mathrm{Nb}$ for which the $Q$ is lowered by more than a factor 10 , to a few million. In order to bring the effective noise temperature of the detector $T_{\text {eff }}$ (as given by Fq. (3)) 
close to the quantum noise limit $T_{\text {quimt }}=h v / k_{b} \approx 5 \times 10^{-8} \mathrm{~K}$, it is important to cool the detector as much as possible and to have a material of very high $Q$. Superconducting alloys, due to their low thermal conductivity are not good materials for this purpose. Copper alloys like $\mathrm{CuBe}, \mathrm{CuAl}, \mathrm{CuSn}$ are better

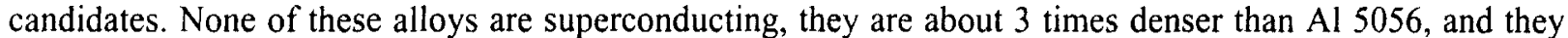
have sound velocities around $4500 \mathrm{~m} \mathrm{~s}^{-1}$ for CuAl, $5300 \mathrm{~m} \mathrm{~s}^{-1}$ for BeCu, and $3600 \mathrm{~m} \mathrm{~s}^{-1}$ for CuSn. The spherical $113 \mathrm{t}$ antenna could in principle be cooled from room temperature to $10 \mathrm{mK}$ in about $3-4$ weeks (Frossati, 1997). For a $1 \mathrm{~ms}$ burst the quantum-limit strain sensitivity would be $h \approx 3 \times 10^{-22}$ (Coccia et al., 1995). Lobo (1994) showed that the second excited quadrupole mode (at double frequency) should have an energy cross-section only 2.5 times lower than the first quadrupole mode, with a loss in strain sensitivity of only a factor 1.6. In fact, this is more sensitive than a spherical antenna with the same fundamental frequency. However, sufficient funding for GRAIL could not be obtained.

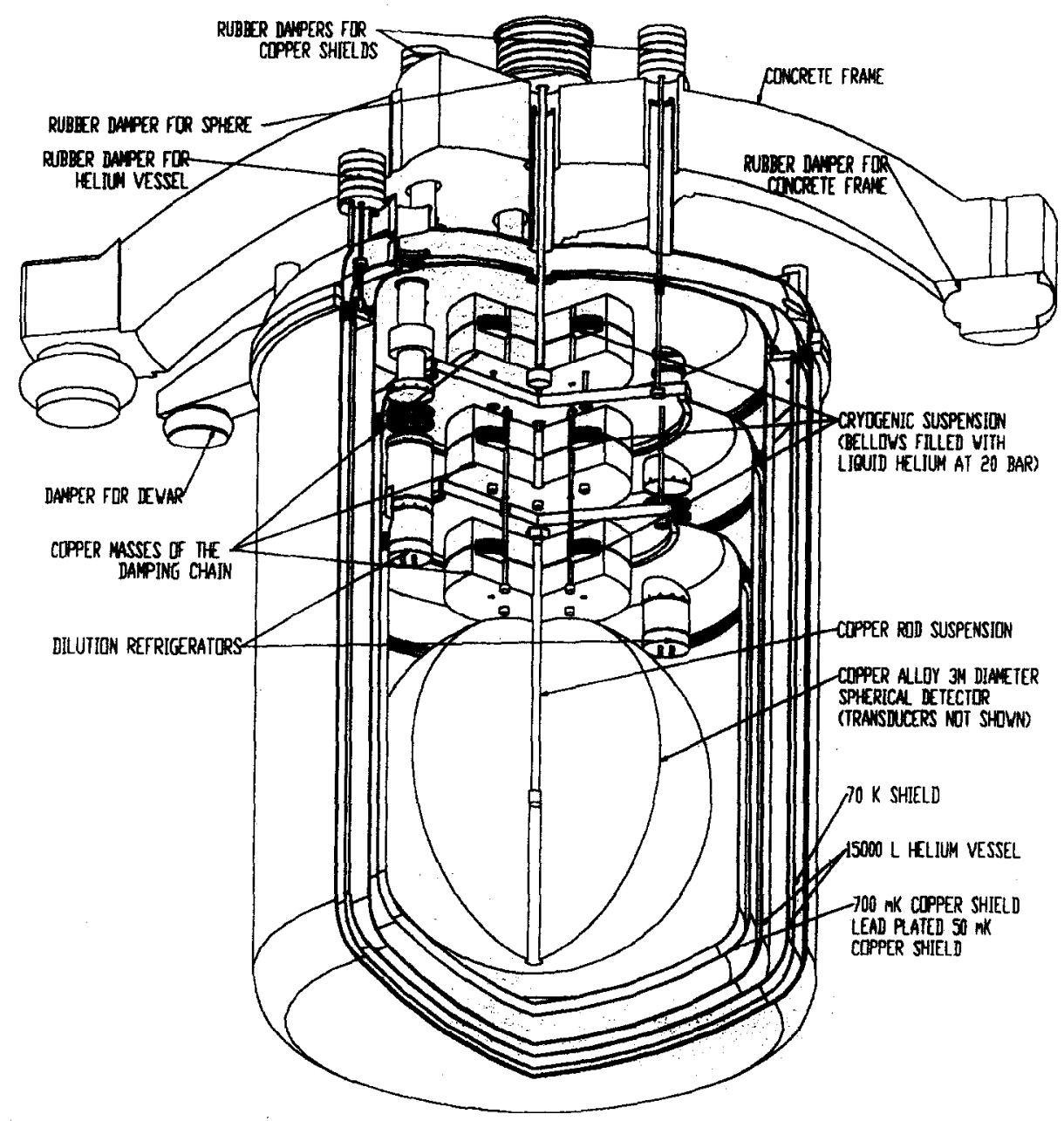

Fig. 1. Cut-away view of the proposed GRAIL resonant cryogenic sphere detector. 


\section{MINIGRAIL}

Currently under discussion is a much smaller, low-cost version of GRAIL, referred to as MiniGRAIL. This is a cryogenic $65 \mathrm{~cm}$ diameter spherical wave antenna made of CuAl (6\%) alloy with a mass of $1150 \mathrm{~kg}$, a resonance frequency of $3250 \mathrm{~Hz}$ and a bandwidth around $230 \mathrm{~Hz}$, operating at $20 \mathrm{mK}$. The primary aim of MiniGRAIL would be to develop the technology necessary to reach sensitivities at least one order of magnitude better than present resonant bar detectors, and at a higher frequency range. It is estimated that MiniGRAIL would achieve a strain sensitivity in the $10^{-20}$ range and a spectral strain amplitude in the $10^{-22} / \sqrt{\mathrm{Hz}}$ range within a few years of operation. Two other detectors of this kind, one in Rome and one in São Paulo, are also in the early planning stages. Operating three identical detectors at widely different locations would strongly increase the chances of gravitational wave detection by searching for coincidences.

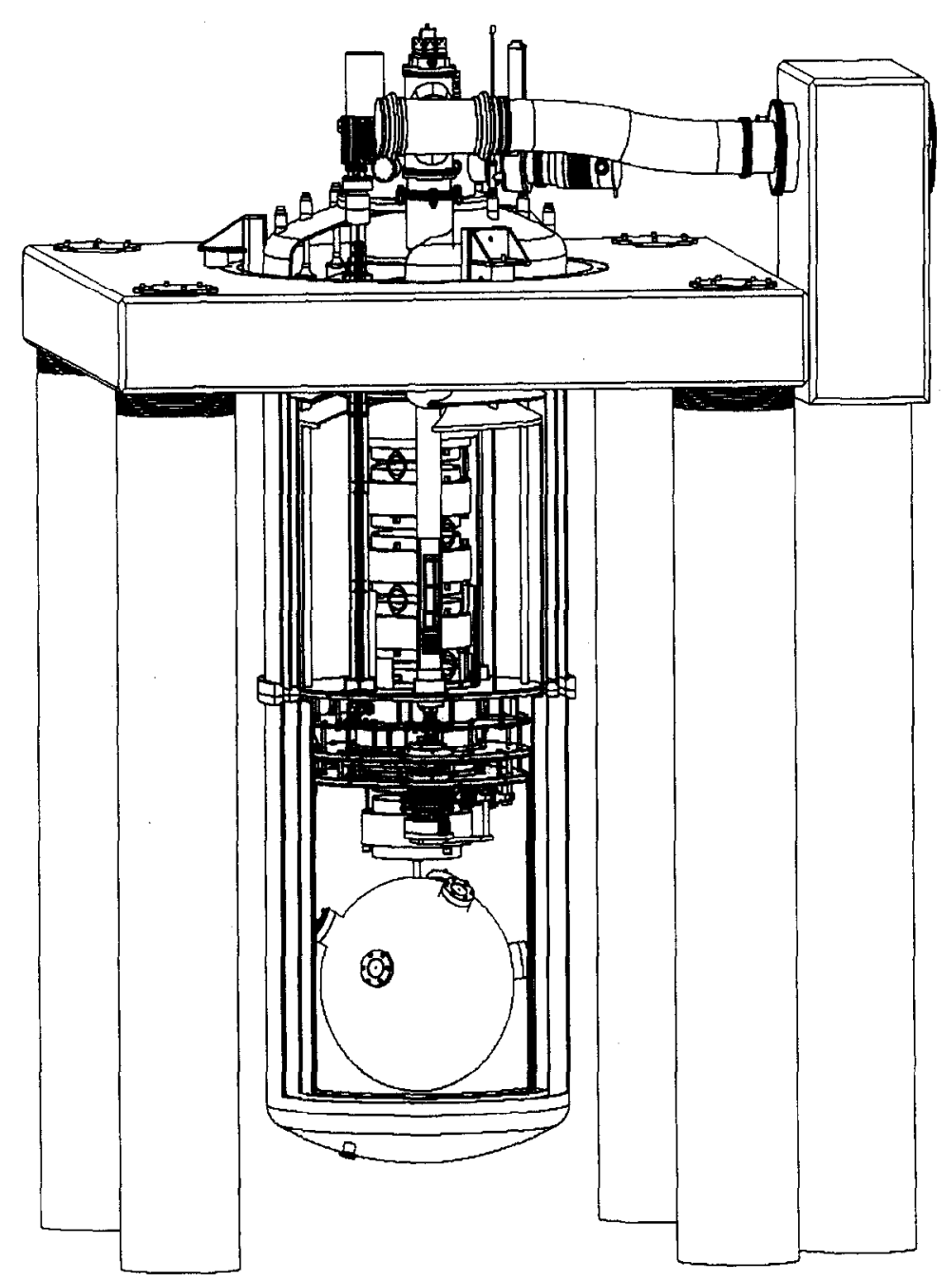

Fig. 2. Cross-sectional view of MiniGRAIL. The height of the cryostat is $3 \mathrm{~m}$. 


\section{CONCLUSION}

Spherical resonant gravitational wave antennas of $100 \mathrm{t}$ mass seem feasible and offer many advantages, such as the relatively modest cost (we estimate it to be about $5 \%$ of that of LIGO) and the high potential sensitivity equal to or better than that of a large interferometer in the high-frequency range. The large mass of these GRAIL detectors lowers the quantum limited spectral strain sensitivity to about $1.3 \times 10^{-24} / \sqrt{\mathrm{Hz}}$. If transducers can be developed with a $30 \%$ bandwidth around both frequencies there will be a good chance of learning something about the shape of gravitational waves, which was only thought possible with interferometric antennas. These possibilities combined with the high directionality of the spheres and their capability of measuring the polarization of the gravitational waves will make spherical detectors indispensable for future gravitational wave astronomy together with the interferometers.

\section{REFERENCES}

Astone, P., C. Cosmelli, S. Frasca, G.V. Pallottino, P. Rapagnani, F. Ricci, M. Bassan, E. Coccia, V. Fafone, Y. Minenkov, I. Modena, A. Moleti, M.A. Papa, P. Bonifazi, R. Terenzi, M. Visco, P. Carelli, A. Marini, G. Mazzitelli, G. Modestino, F. Ronga, L. Votano, and G. Pizzella, The Gravitational Wave Detectors EXPLORER and NAUTILUS, in Proc. First Int. Workshop on "Omnidirectional Gravitational Radiation Observatory", eds. W.F. Velloso, Jr., O.D. Aguiar and N.S. Magalhaes, pp. 3948 (1997).

Blair, D.G., I.S. Heng, E.N. Ivanov, M.E. Tobar, P.J. Turner, and F.J. van Kann, The Perth Niobium Resonant Mass Antenna with Microwave Parametric Transducer, in Proc. First Int. Workshop on "Omnidirectional Gravitational Radiation Observatory", cds. W.F. Velloso, Jr., O.D. Aguiar and N.S. Magalhaes, pp. 27-38 (1997).

Cerdonio, M., M. Bonaldi, D. Carlesso, E. Cavallini, S. Caruso, A. Colombo, P. Falferi, G. Fontana, P.L. Fortini, R. Mezzena, A. Ortolan, G.A. Prodi, L. Taffarello, G. Vedovato, S. Vitale, and J.P. Zendri, The Ultracryogenic Gravitational-Wave Detector AURIGA, Class. Quantum Grav. 14, pp. 1491-1494 (1997).

Coccia, E., V. Fafone, and G. Frossati, On the Design of Ultralow Temperature Spherical Gravitational Wave Detectors, in Proc. First Edouardo Amaldi Conf. on Gravitational Wave Experiments, eds. E. Coccia, G. Pizella, and F. Ronga, World Scientific Publishing Co., Singapore, pp. 463-475 (1995).

Forward, R.L., Multidirectional, Multipolarization Antennas for Scalar and Tensor Gravitational Radiation, Gen. Rel. and Grav. 2, 149-159 (1971).

Frossati, G., A 100 Ton $10 \mathrm{mK}$ Spherical Gravitational Wave Detector, in Proc. First Int. Workshop on "Omnidirectional Gravitational Radiation Observatory", eds. W.F. Velloso, Jr., O.D. Aguiar and N.S. Magalhaes, pp. 163-168 (1997).

Hamilton, W.O., Z.K. Geng, W.W. Johnson, E. Mauceli, S.M. Merkowitz, A. Morse, and N. Salomonson, Performance of the ALLEGRO Detector - and what our Experience Tells us about Spherical Detectors, in Proc. First Int. Workshop on "Omnidirectional Gravitational Radiation Observatory", eds. W.F. Velloso, Jr., O.D. Aguiar and N.S. Magalhaes, pp. 19-26 (1997).

Lobo, A.J. and J.A. Ortega, Interaction Cross-Sections for Spherical Resonant GW Antennae, in Proc. First Edouardo Amaldi Conf. on Gravitational Wave Experiments, eds. E. Coccia, G. Pizella, and F. Ronga, World Scientific Publishing Co., Singapore, pp. 449-454 (1995).

Merkowitz, S.M. and W.W. Johnson, Spherical Gravitational Wave Antennas and the Truncated Icosahedral Arrangement, Phys. Rev. D 51, pp. 2546-2558 (1995).

Wagoner, R.V. and H.J. Paik, Multi-Mode Detection of Gravitational Waves by a Sphere, in Proc. Int. Symposium on "Experimental Gravitation", ed. B. Bertotti, Acc. Naz. dei Lincei, Roma, pp. 257-265 (1977).

Weber, J., Detection and Generation of Gravitational Waves, Phys. Rev. Lett. 117, pp. 307-313 (1960).

Weber, J., Evidence for Discovery of Gravitational Radiation, Phys. Rev. Lett. 22, pp. 1320-1324 (1969). 